\title{
Theoretical Analysis of the Energy Capture in Strictly Bandlimited Ultra-Wideband Channels
}

\author{
Georg Böcherer ${ }^{1}$, Daniel Bielefeld ${ }^{2}$ \\ Institute for Theoretical Information Technology, RWTH Aachen University \\ Aachen, Germany \\ 1 boechererati.rwth-aachen. de \\ 2 bielefeldati.rwth-aachen.de
}

\begin{abstract}
The frequency selectivity of wireless communication channels can be characterized by the delay spread $D_{s}$ of the channel impulse response. If the delay spread is small compared to the bandwidth $W$ of the input signal, that is, $D_{s} W \approx 1$, the channel appears to be flat fading. For $D_{s} W \gg 1$, the channel appears to be frequency selective, which is usually the case for wideband signals. In the first case, small scale synchronization with a precision much higher than the sampling time $T=1 / W$ is crucial to guarantee the maximum capture of energy at the receiver. In this paper, it is shown by analytical means that this is different in the wideband regime. Here synchronization with a precision of $T$ is sufficient and small scale synchronization cannot further increase the captured energy at the receiver. Simulation results show that this effect already occurs for $W>50 \mathrm{MHz}$ for the IEEE 802.15.4a channel model.
\end{abstract}

\section{INTRODUCTION}

Ultra-wideband communication offers the attractive possibility to achieve high data rates with low transmission power and is a candidate for the air interface of next generation wireless personal area networks [1].

The transmitted ultra-wideband signal is usually subject to frequency selective fading due to multi-path propagation. In literature, the involved channel has been modeled in different ways. Physically inspired approaches aim to give a tapped delay-line model of the propagation environment in terms of a time-continuous impulse response which consists of the superposition of arbitrarily delayed and scaled versions of the signal emitted by the sender. In [2], the authors refer to the number of delayed and scaled versions assumed at the receiver as the diversity level of the receiver and they observe that with an increasing diversity level, the amount of captured energy increases.

The authors in [3] use a more general model. They assume a strictly bandlimited transmitted signal and model the channel by taps as observed by the receiver after appropriate lowpass filtering and sampling of the received signal with a sampling time according to the sampling theorem. The obtained timediscrete channel model consists of a finite number of channel taps. A similar model is used in [4], where the sampling time is referred to as the system resolution. In these models, the observed channel taps depend heavily on the chosen time instants of the sampling process. For different timing offsets between transmitter and receiver, corresponding to different sampling time instants, the receiver might observe different realizations of the channel, although the physical channel remains the same. In the following, the different realizations of channel taps for the same physical channel in the timediscrete model are referred to as channel candidates. For a low number of channel taps, normally corresponding to a low signal bandwidth, the unknown timing offset can lead to a significant degradation of the amount of energy that can be captured by the receiver, since different observed channel candidates have in general different channel gains. To account for this in practical communication systems, synchronization algorithms are employed to conduct a precise estimate of the timing offset and to synchronize the time references of transmitter and receiver [5].

In this paper, we analytically investigate the problem of different channel candidates in the wideband limit. We show that in this case, all channel candidates have the same channel gain if a large scale synchronization in the order of a sampling time interval is assumed. This is not obvious: although the system resolution increases with increasing bandwith, also the degrees of freedom of the effective channel increases linearly with the bandwidth [3], which could cancel out the effect of increasing resolution. This is illustrated in Figure 1 Our result implies that for a large bandwidth of the input signal, no additional term in the discrete time channel model of [3] is necessary to consider the delay between transmitter and receiver, since it would not make the model more representative.

To validate our results and to obtain numerical values for the amount of the performance degradation for a realistic scenario, simulations were conducted using the IEEE 802.15.4a channel model. The results indicate that for a narrow communication bandwidth $(\leq 16 \mathrm{MHz})$, a missing small scale synchronization can lead to an energy capture degradation of more than $25 \%$. For increasing bandwidth, the degradation vanishes. If we interpret the corresponding number of considered channel taps as the diversity level of the receiver, this result is in accordance with the observation in [2].

The remainder of the paper is organized as follows. In Section III, a mathematical description of the considered problem is given. Based on this, we investigate the problem analytically in Section III In Section IV, we describe the simulation procedure and discuss the obtained results.

\section{SySTEM MODEL}

We consider a linear time-invariant (LTI) system that is specified by a complex continuous time impulse response $h$, 


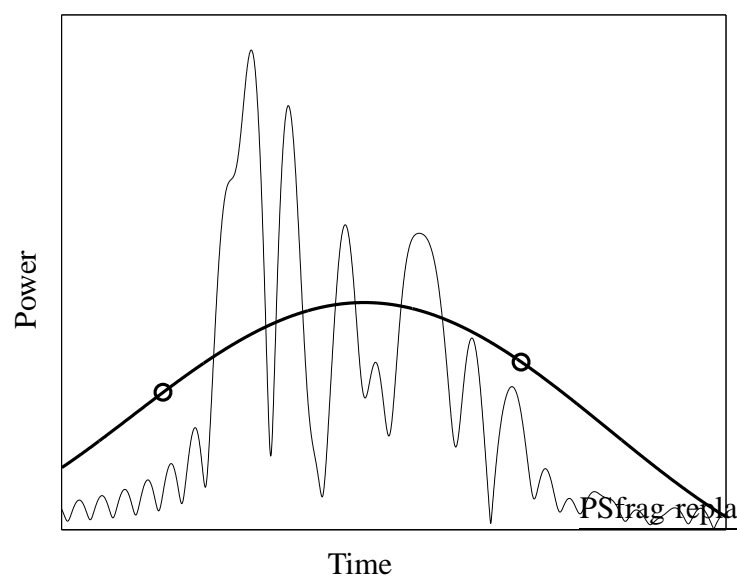

(a) Sampling in narrowband

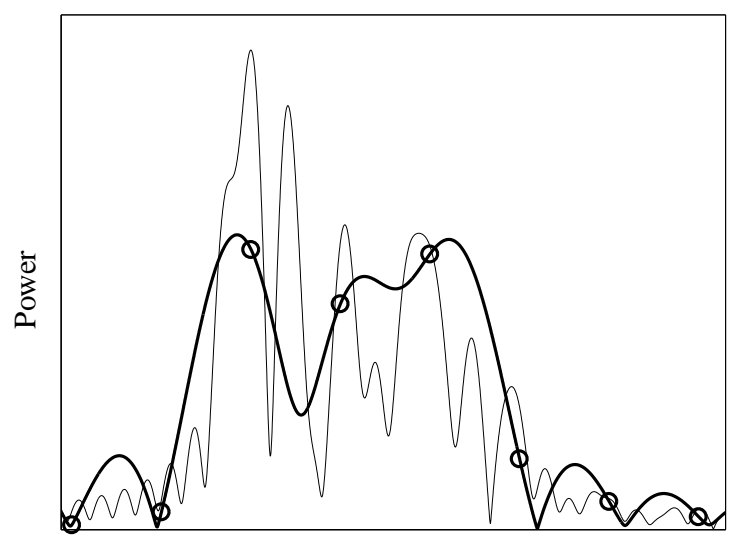

Time

(b) Sampling in wideband

Fig. 1. An impulse response of bandwidth $128 \mathrm{MHz}$ (thin line) is in subfigure (a) filtered by a lowpassfilter of bandwidth $8 \mathrm{MHz}$ (bold line), and then sampled (circles). In subfigure (b), the same impulse response is filtered by a lowpassfilter of bandwidth $64 \mathrm{MHz}$ and then sampled. As can be observed, both system resolution and degrees of freedom of the filtered impulse response increase with increasing filter bandwidth.

which we assume in the following to be in $\mathcal{L}^{2}(\mathbb{R})$, the space of energy limited signals. The impulse response is of finite length, that is, there exists some delay spread $D_{s}>0$ such that

$$
\forall \tau \notin\left[0, D_{s}\right]: h(\tau)=0 .
$$

The input-output (I/O) relation between a transmitted signal $s$ and the corresponding received signal $y$ is then given by the convolution

$$
y(t)=\int_{-\infty}^{\infty} h(\tau) s(t-\tau) \mathrm{d} \tau .
$$

If the input signal is bandlimited to $W$, then the output signal is also bandlimited to $W$ and we can define the effective impulse response $h_{T}$ of the LTI system as the result of lowpass filtering $h$ by a unit-gain lowpass filter of bandwidth $W=1 / T$. It is important to note that the effective impulse response has no longer a finite delay spread, so we have in general $h_{T}(\tau) \neq 0$ for $\tau \in \mathbb{R}$. Applying the sampling theorem, the system can be described by the discrete time I/O relation given by

$$
y[k]=\sum_{l=-\infty}^{\infty} h_{T}[l] s[k-l] .
$$

Although we have in general $h[l] \neq 0$ for $l \in \mathbb{Z}$, it is infeasible in practice to treat an infinite number of channel taps. We therefore truncate the $\mathrm{I} / \mathrm{O}$ relation and consider only a finite number $L$ of channel taps. The truncated I/O relation is given by

$$
y[k]=\sum_{l=0}^{L-1} h_{T}[l] s[k-l] .
$$

For the number $L$, an appropriate number corresponding to the delay spread $D_{s}$ should be chosen.

The relation (4) is a standard model for frequency selective channels, see [6]. The authors of [3] modeled an UWB indoor environment by (4) and based their measurements on this model.

We will take a closer look at how (4) is related to (2). The discrete time I/O relation results from two sampling processes, one at the sender and one at the receiver. We assume that the drift between the two sampling clocks is already compensated. What remains is a timing offset between the two sampling clocks. We model this offset by including an arbitrary timing offset $d$ between sender and receiver in our model. The timing offset $d$ can be written as

$$
d=\left\lfloor\frac{d}{T}\right\rfloor T+\delta
$$

with $\delta \in[0, T)$. We assume that the system has already performed a large scale acquisition of the timing and knows $\lfloor d / T\rfloor$. Without loss of generality, we can therefore assume $\lfloor d / T\rfloor=0$. The samples are then given by

$$
y[k]=y(k T-\delta), h_{T}[l]=h_{T}(l T-\delta), s[k]=s(k T)
$$

where $t$ is the timing reference at the sender and $t-\delta$ is the timing reference at the receiver. For a certain timing offset $\delta$, the channel candidate, which will be estimated by the receiver, is given by

$$
\tilde{h}_{T}^{(\delta)}[l]= \begin{cases}h_{T}(l T-\delta), & 0 \leq l<L \\ 0, & \text { otherwise. }\end{cases}
$$

We set the number of channel taps $L$ equal to

$$
L=\left\lfloor\frac{D_{s}}{T}\right\rfloor \text {. }
$$

This assignment is to certain point arbitrary. The trade-off between system performance and complexity may lead to other values for $L$ in practice.

The small scale timing synchronization at the receiver now consists in finding $\delta$ such that the corresponding channel candidate (7) used in (4) represents (2) in the best possible way. To quantify the quality of a certain channel candidate 
(7), we look at the overall channel gain $\left\|\tilde{h}_{T}^{(\delta)}\right\|^{2}$, which is given by

$$
T\left\|\tilde{h}_{T}^{(\delta)}\right\|^{2}=\sum_{l=0}^{L-1} T\left|h_{T}(l T-\delta)\right|^{2} .
$$

Depending on the bandwidth $W$ and the corresponding sampling time $T=1 / W$, the channel gain varies for different $\delta \in[0, T)$. As we will see in the following sections, the variance of the channel gain goes to zero for $W$ tending to infinity.

\section{ANALYSIS}

For the family of channel candidates (7), we state the limit property of the overall channel gain as a theorem.

Theorem 1. Let the channel candidates as given in (7) be of bandwidth $W=1 / T$. Then, for every $\delta \in[0,1 / W)$, the overall channel gain converges to the maximum value given by $\left\|\tilde{h}_{T}^{(\delta)}\right\|^{2}=\|h\|^{2}$ when the bandwidth $W$ tends to infinity.

Before we can give the proof of the theorem, we state Plancherel's Theorem. It can be found e.g. in [7].

Theorem 2. (Plancherel) For any $x \in \mathcal{L}^{2}(\mathbb{R})$ (the Hilbert space of energy limited signals), there exists a function $\mathrm{F}\{x\} \in$ $\mathcal{L}^{2}(\mathbb{R})$, such that

$$
\lim _{T \rightarrow \infty} \int_{-\infty}^{\infty}\left|\mathrm{F}\{x\}(f)-\int_{-\frac{T}{2}}^{\frac{T}{2}} x(t) e^{-j 2 \pi f t} \mathrm{~d} t\right|^{2} \mathrm{~d} f=0
$$

and

$$
\lim _{W \rightarrow \infty} \int_{-\infty}^{\infty}\left|x(t)-\int_{-\frac{W}{2}}^{\frac{W}{2}} \mathrm{~F}\{x\}(f) e^{j 2 \pi f t} \mathrm{~d} f\right|^{2} \mathrm{~d} t=0 .
$$

The function $\mathrm{F}\{x\}$ is called the Fourier transform of $x$.

We assume in the following that for all functions $x$ of interest, the integral

$$
\int_{-\infty}^{\infty} x(t) e^{-j 2 \pi f t} \mathrm{~d} t
$$

exists. The Fourier transform $\mathrm{F}\{x\}$ is then given by (12). We will need the following convention in the proof of Theorem 1 We define the effective impulse response $h_{T}$ point-wise by the inverse Fourier transform of $\mathrm{F}\left\{h_{T}\right\}$ given by

$$
h_{T}(\tau)=\int_{-\infty}^{\infty} \mathrm{F}\left\{h_{T}\right\}(f) e^{j 2 \pi f \tau} \mathrm{d} f .
$$

It can easily be shown that with this definition, $h_{T}$ is continuous.
Proof of Theorem 17. The energy of $\tilde{h}_{T}^{(\delta)}$ is bounded from above by

$$
\begin{aligned}
\|h\|^{2} & \geq\left\|h_{T}\right\|^{2} \\
& \geq T\left\|\tilde{h}_{T}^{(\delta)}\right\|^{2} \\
& =\sum_{l=0}^{\left\lfloor\frac{D_{s}}{T}\right\rfloor-1} T\left|h_{T}(l T-\delta)\right|^{2}
\end{aligned}
$$

where $\delta \in[0, T)$. We show that (16) converges to $\|h\|^{2}$ for $T \rightarrow 0$. Calculating first the limit for $\delta \rightarrow 0$ and then the limit for $T \rightarrow 0$ would lead to the desired result, but this implies not necessarily that (16) converges to the same value if $\delta$ and $T$ tend jointly to zero along any path with $\delta<T$, but it is the latter we have to show.

We introduce the auxiliary parameter $T^{\prime}$ and write

$$
\sum_{l=0}^{\left\lfloor\frac{D_{s}}{T^{\prime}}\right\rfloor-1} T^{\prime}\left|h_{T}\left(l T^{\prime}-\delta\right)\right|^{2} .
$$

The introduction of the parameter $T^{\prime}$ can be interpreted as a separation of the sampling process from the lowpass filtering process.

We now approximate $\|h\|^{2}$ in three steps.

1. Approximation. From Theorem 2 it follows that the integral

$$
\int_{-\infty}^{\infty}\left|h(\tau)-h_{T}(\tau)\right|^{2} \mathrm{~d} \tau
$$

goes to 0 for $T \rightarrow 0$. Since the support of $h$ is $\left[0, D_{s}\right]$, also

$$
\int_{0}^{D_{s}}\left|h(\tau)-h_{T}(\tau)\right|^{2} \mathrm{~d} \tau
$$

goes to 0 . For any $v, w \in \mathcal{L}^{2}(\mathbb{R})$, the reverse triangular inequality states that

$$
|\|v\|-\|w\|| \leq\|v-w\| .
$$

It follows that for any $\epsilon_{1}>0$ there exists a $T_{1}$ such that

$$
\left.\left|\int_{0}^{D_{s}}\right| h(\tau)\right|^{2} \mathrm{~d} \tau-\int_{0}^{D_{s}}\left|h_{T}(\tau)\right|^{2} \mathrm{~d} \tau \mid<\epsilon_{1}
$$

for all $T<T_{1}$.

2. Approximation. For every $\delta \in \mathbb{R}$, let $h_{T}^{(\delta)}$ denote the translation of $h_{T}$ defined by $h_{T}^{(\delta)}(\tau)=h_{T}(\tau-\delta), \tau \in \mathbb{R}$. According to [8, Theorem 9.5], the mapping

$$
\delta \mapsto h_{T}^{(\delta)}
$$

is uniformly continuous in $\mathcal{L}^{2}(\mathbb{R})$. Together with the reverse triangular inequality (19), this implies that for every $\epsilon_{2}>0$, there exists a $\delta_{1}$ such that

$$
\left.\left|\int_{0}^{D_{s}}\right| h_{T}(\tau)\right|^{2} \mathrm{~d} \tau-\int_{0}^{D_{s}}\left|h_{T}(\tau-\delta)\right|^{2} \mathrm{~d} \tau \mid<\epsilon_{2}
$$

for all $\delta<\delta_{1}$. 
3. Approximation. Since $h_{T}$ is continuous and since we consider it over a compact interval, the term

$$
\sum_{l=0}^{\left\lfloor\frac{D_{s}}{T^{T}}\right\rfloor-1} T^{\prime}\left|h_{T}\left(l T^{\prime}-\delta\right)\right|^{2}
$$

is equal to the Riemann sum of $\left|h_{T}(\tau-\delta)\right|^{2}$ over the interval $\left[0, D_{s}\right]$. For $T^{\prime} \rightarrow 0$, the Riemann sum converges to the integral. Therefore, for every $\epsilon_{3}>0$, there exists a $T_{1}^{\prime}$ such that

$$
\left.\left|\int_{0}^{D_{s}}\right| h_{T}(\tau+d)\right|^{2} \mathrm{~d} \tau-\sum_{l=0}^{\left\lfloor\frac{D_{s}}{T^{*}}\right\rfloor-1} T^{\prime}\left|h_{T}\left(l T^{\prime}-\delta\right)\right|^{2} \mid<\epsilon_{3}
$$

for all $T^{\prime}<T_{1}^{\prime}$. We define $\epsilon_{0}=\epsilon_{1}+\epsilon_{2}+\epsilon_{3}$ and $T_{0}=\min \left\{T_{1}, T_{1}^{\prime}, \delta_{1}\right\}$. Combining the three approximations, we have shown that for every $\epsilon_{0}>0$, there exists a $T_{0}$ such that

$$
\left.\left|\|h\|^{2}-\sum_{l=0}^{\left\lfloor\frac{D_{s}}{T^{\prime}}\right\rfloor-1} T^{\prime}\right| h_{T}\left(l T^{\prime}-\delta\right)\right|^{2} \mid<\epsilon_{0}
$$

for all $T, T^{\prime}, \delta<T_{0}$. This result is also valid for $T^{\prime}=T$ and $\delta$ and $T$ jointly tending to zero with $0 \leq \delta<T$. This concludes the proof.

For a further discussion of this result we refer to Section IV

\section{Simulation}

We simulate the effect of the timing offset $\delta$ onto the overall gain of channel candidates by calculating $\left\|\tilde{h}_{T}^{(\delta)}\right\|^{2}$ for increasing bandwidths $W$. As a data set, we use 100 impulse responses according to the IEEE 802.15.4a UWB channel model defined in [9] by using the MATLAB script uwb sv eval ct 15 4a.m, which is also provided in [9]. The generated impulse responses have a normalized delay spread of $D_{s}=279 \mathrm{~ns}$. Since the data set already comes in digital form and since we are limited to digital signal processing in simulation, it is difficult to compare all possible channel candidates for $\delta \in[0, T), \delta$ continuous. We therefore resort to the following. We consider a generic all digital receiver that has a small scale timing synchronizer with a time resolution of $T / 4$. With this receiver in mind, we compare the channel candidates for every impulse response $h^{(i)}$ from the data set and every considered bandwidth $W=1 / T$ in the following way.

Step 1. We lowpass filter $h^{(i)}$ to obtain $h_{T}^{(i)}$.

Step 2. We randomly generate a small timing offset $\varepsilon$ uniformly distributed over $[0, T / 4)$. The generic receiver in mind will not resolve $\varepsilon$.

Step 3. For $m=0, \ldots, 3$, we consider the sequence

$$
h_{T}^{(i)}\left(l T-\varepsilon-\frac{m T}{4}\right)
$$

and calculate the channel gain of the truncated impulse response to

$$
\left\|\tilde{h}_{T}^{(i, m)}\right\|^{2}=\max _{k} \sum_{l=0}^{\left\lfloor\frac{D_{s}}{T}\right\rfloor-1} T\left|h_{T}^{(i)}\left(k T+l T-\varepsilon-\frac{m T}{4}\right)\right|^{2} .
$$

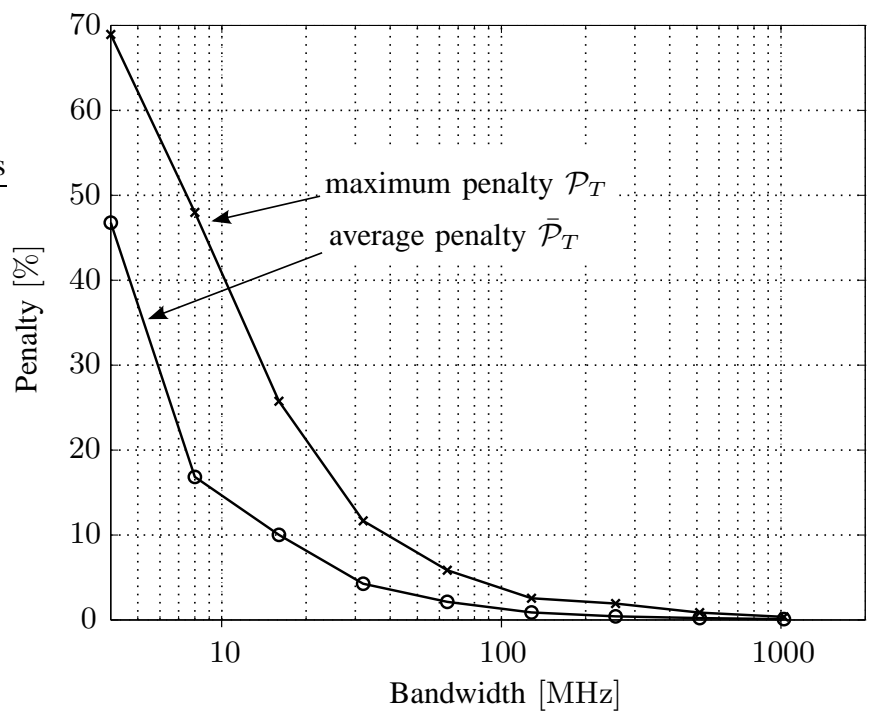

Fig. 2. The average relative penalty $\overline{\mathcal{P}}_{T}$ and the maximum relative penalty $\mathcal{P}_{T}$ for timing acquisition without small scale timing synchronization as a function of the bandwidth $W=1 / T$.

At the receiver, the maximization over $k$ corresponds to an energy based large scale timing acquisition. The maximum relative channel gain loss when synchronizing without a small scale timing synchronizer is given by

$$
\mathcal{P}_{T, \max }^{(i)}=1-\frac{\min _{m}\left\|\tilde{h}^{(i, m)}\right\|^{2}}{\max _{m}\left\|\tilde{h}^{(i, m)}\right\|^{2}} .
$$

We assess the simulation results by considering both the maximum relative performance penalty and the average relative performance penalty given by

$$
\mathcal{P}_{T}=\max _{i} \mathcal{P}_{T, \max }^{(i)} \quad \text { and } \quad \overline{\mathcal{P}}_{T}=\frac{1}{100} \sum_{i=1}^{100} \mathcal{P}_{T, \max }^{(i)}
$$

as functions of the sampling time $T=1 / W$. In Table \ we provide the simulation results consisting of the corresponding values for the bandwidth, the worst case penalty, the average penalty, and the number of channel taps. As can be seen from Figure 2. both the worst case penalty and the average penalty decrease monotonically with an increasing bandwidth. Both curves converge to 0 , which corresponds to our analytic result from the previous section.

We observe that, roughly for $L=\left\lfloor D_{s} W\right\rfloor>10$, synchronizing with a resolution of $T$ is sufficient in terms of channel gain, since the maximum penalty is smaller than $5 \%$. On the other hand, in a narrowband scenario with $L=\left\lfloor D_{s} W\right\rfloor<4$, the penalty due to a missing small scale synchronizer can be over $50 \%$.

\section{CONCLusions}

We have shown that for communications strictly bandlimited to $W$ over an LTI channel with delay spread $D_{s}$, the truncated discrete time channel model

$$
y[k]=\sum_{l=0}^{L-1} h[l] x[k-l], \quad L \approx\left\lfloor D_{s} W\right\rfloor
$$


TABLE I

SimULATION RESULTS FOR $D_{s}=279 \mathrm{~ns}$

\begin{tabular}{ll|rrrrrrrrr}
\hline$W$ & {$[\mathrm{MHz}]$} & 4 & 8 & 16 & 32 & 64 & 128 & 256 & 512 & 1024 \\
$\mathcal{P}$ & {$[\%]$} & 68.9 & 48.0 & 25.8 & 11.7 & 5.8 & 2.5 & 1.9 & 0.9 & 0.4 \\
$\overline{\mathcal{P}}$ & {$[\%]$} & 46.8 & 16.8 & 10.0 & 4.3 & 2.1 & 0.9 & 0.4 & 0.2 & 0.1 \\
$L=\left\lfloor D_{s} W\right\rfloor$ & 1 & 2 & 4 & 8 & 17 & 35 & 71 & 142 & 285 \\
\hline
\end{tabular}

is robust against unknown timing offsets $\delta \in[0,1 / W)$ between sender and receiver in the limit when the bandwidth $W$ of the considered system goes to infinity. A simulation shows that this theoretic result is valid for the IEEE 802.15.4a channel model for $W>50 \mathrm{MHz}$. As a receiver design criterion, this means that small scale synchronization with a precision higher than the sampling time $T=1 / W$ is not necessary for UWB communication systems. As an extension of our work, it may be of interest to investigate if the robustness against the small scale timing offset $\delta$ remains when drift compensation, large scale timing acquisition and channel estimation in the design of a wideband receiver are jointly considered.

\section{ACKNOWLEDGMENT}

This work was partly supported by the Deutsche Forschungsgemeinschaft (DFG) project UKoLoS (grant MA 1184/14-1) and the UMIC excellence cluster of RWTH Aachen University.

\section{REFERENCES}

[1] H. Arslan, Z. Chen, and M.-G. Di Benedetto, Eds., Ultra Wideband Wireless Communication. Hoboken, New Jersey: Wiley-Interscience, 2006.
[2] M. Win and R. Scholtz, "On the energy capture of ultrawide bandwidth signals in dense multipath environments," IEEE Commun. Lett., vol. 2, no. 9 , pp. $245-247,1998$.

[3] U. Schuster and H. Bolcskei, "Ultrawideband channel modeling on the basis of information-theoretic criteria," IEEE Trans. Wireless Commun., vol. 6, no. 7, pp. 2464-2475, 2007.

[4] I. Telatar and D. Tse, "Capacity and mutual information of wideband multipath fading channels," IEEE Trans. Inf. Theory, vol. 46, no. 4, pp. 1384-1400, 2000.

[5] H. Meyr, M. Moeneclaey, and S. A. Fechtel, Digital Communication Receivers. John Wiley \& Sons, Inc, 1998.

[6] D. Tse and P. Viswanath, Fundamentals of Wireless Communication. Cambridge University Press, 2005.

[7] R. E. A. C. Paley and N. Wiener, Fourier Transforms in the Complex Domain, ser. Colloquium Publications. New York: American Mathematical Society, 1934, vol. XIX.

[8] W. Rudin, Real and Complex Analysis, 3rd ed. McGraw-Hill Publishing Co., 1987.

[9] A. F. Molisch, K. Balakrishnan, C. C. Chong, S. Emami, A. Fort, J. Karedal, J. Kunisch, H. Schantz, U. Schuster, and K. Siwiak, "IEEE 802.15.4a channel modelfinal report," Tech. Rep., $2004 . \quad$ [Online]. Available: http://www.ieee802.org/15/pub/04/15-04-0662-00-004a-channel-model-final-report-r1.p 